\title{
Rhombohedral Super Hetero Epitaxy of Cubic SiGe on Trigonal c-plane Sapphire
}

\author{
Sang H. Choi \\ Adam J. Duzik \\ NASA Langley Research Center \\ Hampton, VA 23681-2199
}

\begin{abstract}
New rhombohedral super-hetero-epitaxy technology was developed at NASA. This epitaxy technology enables the growth of unprecedented cubic-trigonal hybrid single crystal structures with lattice match on sapphire $\left(\mathrm{Al}_{2} \mathrm{O}_{3}\right)$ substrates, hence with little strain and very few defects at the interface.
\end{abstract}

\section{Summary}

Today's semiconductor industry is based on two bandgap engineering crystal alloy models: cubic and hexagonal crystal structure constraints. New hybrid bandgap engineering based on rhombohedral super-hetero-epitaxy technology was developed by a team of scientists at NASA Langley Research Center in the late 2000s. This technology enables the growth of unprecedented cubic-trigonal hybrid single crystal structure alloys with lattice match on sapphire $\left(\mathrm{Al}_{2} \mathrm{O}_{3}\right)$ substrates. This affords minimal strain, and minimal interfacial defects. As a result of this development, a new hybrid bandgap engineering model was generated allowing for thousands of new semiconductor structures to be fabricated (see the figure below). This technology received a prestigious R\&D100 award (US) in 2009 and Solar Industry Award (EU) in 2010, and awarded 10 patent and patent applications.

Heteroepitaxy of cubic or zinc blende structures on a trigonal C-plane sapphire substrate presents a considerable challenge in matching lattice constants between the substrate and the host materials. The recently patented Rhombo-Trigonal epitaxy method enables growth of a high-quality single crystalline [111] silicon-germanium layer on a trigonal C-plane sapphire substrate (1-4). However, the process itself requires an unusually high operational temperature to energize the cubic structure enough to be rotated, and deformed into a rhombohedron anchored on a trigonal structure of sapphire. A new mechanism to grow such a rhombohedral epitaxy at a lower substrate temperature has been implemented by energizing the atoms in flux allowing for the reduction of the substrate temperature to a moderate level. The sufficiently energized atoms provide the essential energy needed for the rhombohedral epitaxy process, which deforms the original cubic crystalline structure into a rhombohedron by physically aligning the crystal structure of both materials at a lower substrate temperature. Typically, a strained layer of epitaxy is developed as a precursor structure in order to 
build a certain thickness level of epitaxy. The newly developed mechanism drastically differs from conventional methods.

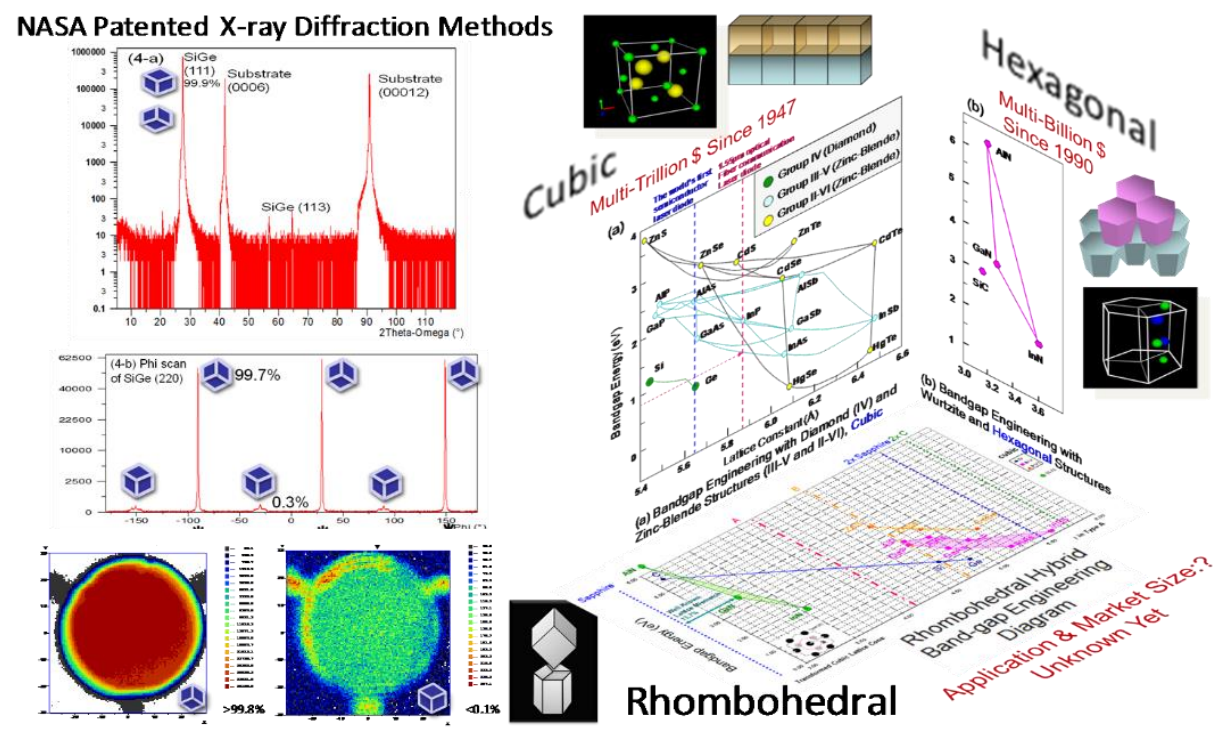

References:

(1) Yeonjoon Park, Glen C. King, Sang H. Choi, "Rhombohedral epitaxy of cubic SiGe on trigonal c-plane sapphire", Journal of Crystal Growth, 310, pp. 2724-2731 (2008)

(2) Yeonjoon Park, Sang H. Choi, Glen C. King, "Silicon germanium semiconductive alloy and method of fabricating same", United States Patent \# 7341883 B2 (2008)

(3) Yeonjoon Park, Sang H. Choi, James R. Elliott, "Hybrid bandgap engineering for super-hetero-epitaxial semiconductor materials, and products thereof", US Patent \#822767 (2012)

(4) Yeonjoon Park and Sang H. Choi, "Beyond The Silicon-Based Chips", Feature Article of Compound Semiconductor Magazine, Vol. 16, No. 3, pp 18-21, April-May, (2010)

Presenting author's email: $\quad$ sang.h.choi@nasa.gov 